\title{
Trends in Biomedical Research
}

\section{The role of wild dreams in living objects}

\author{
Martin Vlček* \\ Researcher, Working in eFunctionality, a non-profit research group, Czech Republic
}

\begin{abstract}
Based on economic modeling in living systems, described e.g. in 3) till 5), the potential role of dreams in living objects is examined. It appears that their role is irreplaceable and serves as a screening mechanism. Everything takes place in the context of increasing the object's existence - see also 1) and 2).

In light of the findings, the question is whether dreams can be used for objective diagnosis. The article concludes that this is possible, but after further studies.
\end{abstract}

\section{Introduction}

The living object is understood in the following as an organized group of elements whose organization is extremely (!) Specific. It contains a mechanism for managing its own existence.

The object tries to structure the surrounding activity in its inexhaustible character into individual repetitive groups of partial activities that are characterized by some influence on its existence. These clusters are hereinafter referred to as events. They have one common cause and the object registers them in this unity.

In its own language of statements, similar to the language of mathematics, it writes the event object to memory structures (PS). Eventually remembered and eventually generated events are then involved in other activity structures until the overall reaction of the living object to the surroundings arises. All in the spirit of increasing the rate of its existence.

Surroundings and its events, however, are undergoing constant development, and so the object must constantly adjust their PS. It uses a special apparatus, which includes a dream [1-5].

\section{Event}

All activity taking place (above all) in the vicinity of the building creates one aggregate activity - event - based on the causal context of the grouping of activities. These groupings are repeated to varying degrees each time the cause occurs, but they are never the same. It is the object of the object to create an identification apparatus for each cause, which, even in an incomplete grouping of activities, can generate an event and thus identify the underlying cause. The event itself is zero or one, whether or not it occurred. There are no other values or options. $:$

All the activity of the object in the direction of its surroundings or identification of important phenomena, eventually their prediction and influence on them, is based on the mechanism of event generation.

\section{Existence of an object}

Although the concept of the existence of an object may seem a useless philosophical category, it is in the background of the functioning of every living object at every moment. The whole principle of its operation is based on a simple law: any activity must aim at increasing its existence. The object must therefore always have information on this rate.

Existence is a function of the tendency of the elements of an object to leave it. There is also a tendency to abandon any existing internal structures as a source of helpful information. The part of the management responsible for evaluating the existence is based primarily on the distribution of satisfaction of the elements at the object boundary.

Long-term monitoring of the systems shows that in the case of the economic model, namely the US stock market, the existence rate is monitored on the turbulence in the real estate segment. American companies - and certainly not only them - characterized by the dynamics of their shares create a living object in their relationships $(-)$.

\section{Examination of PS}

The statement and its PS have slightly different properties in the living object than the mathematical statement. You could say it is his superstructure. Every statement has a counterpart around the object. His output is very similar to this counterpart, and the degree of this form is also a measure of its truthfulness. The event generated by the PS is similar to the corresponding nearby event.

But besides this truth, the statement has another essential property - and that is its form with mathematical statements. Its PS, ie the statement-generating structure in terms of its output and imitation of its counterpart in the neighborhood, must still be formally properly built. It must be based on primitive notions and stand above axioms or other formally correct statements.

\section{Truthfulness of the statement}

The more truthful the statement is, the greater is the existence of an object as a result of a change in its surroundings caused by its output - an event.

${ }^{\star}$ Correspondence to: Martin Vlček, Researcher, Working in eFunctionality, a non-profit research group, Czech Republic, Tel: +420737164850; E-mail: martin1408@post.cz

Received: October 02, 2019; Accepted: October 22, 2019; Published: October 25,2019 


\section{Correct statement}

A statement is formally correct if it is ultimately based on primitive concepts and axioms. In other words, its activity may be the same if it somehow reflects environmental influences, or on the contrary, it is based only on internal stimuli.

Because the output of the statement somehow imitates changes in the surroundings that are essential to the existence of the object and which it understands in other words because it has PS for them, the correct statement is based only on "understandable" changes in the surroundings. These are in any case changes that repeat and change the existence of an object. These are therefore changes that are plausible from the point of view of the object, in other words repeatedly essential - for the existence.

In summary, formal accuracy ensures that PS does not reflect an isolated event, even if it strongly affects the existence of an object.

\section{Wild dream}

The object of the object is to make the event in PS as close as possible to the corresponding event in the neighborhood. Given the definition of an event, this similarity is only expressable by the correspondence of the occurrence of the two events. In their mutual frequency.

For the work of the object it is important that the PS can work independently of the surrounding environment. Therefore, the object must have a mechanism to evaluate this credibility when disconnected. This mechanism is a dream.

It detaches the object from the surroundings and compares PS activity. If the activity is similar to the activity during waking, in other words PS generates events similarly in both situations, the influence of the environment on the PS activity is small.

And the measure of this smallness is also a measure of the credibility of the work of the PS.

Ideally, if it is the same activity, it means that everything necessary is stored in the PS. And that is the mechanism of the dream. If the dream experience of (different) PS is similar to what is experienced in waking, if the dream is mundane, normal, real, it means that there is no need to change PS, that there is similar activity in both states.

But if the dream is 'wild', it makes no sense that it differs from normal, recent activities and that the PS needs to be adjusted.

\section{Evidence apparatus}

The object has an apparatus similar to a mathematical apparatus for evaluating the truth of the PS. Above all, they are some kind of "primitive concepts" and "axioms".

This apparatus is based on the fact that it is a living object and it is governed by general, uniform principles of functioning.
It is an organization of elements where their certain groups have corresponding functions in the system of living system management. These are standard, necessary parts of the living system - mathematically speaking of primitive terms - and standard, organizing relationships between these groups - axioms.

It is natural that the object does not examine this structure of relations between parts $(-)$, which in turn defines it. However, it examines all other structures to be newly memorized, ie stored next to existing defining structures - axioms. The examined WG is then a structure of relations between primitive concepts, or between new WGs.

\section{Dream}

A dream is a mechanism that has two basic characteristics. First, the object is disconnected from reality, ie surroundings. On the other hand, the PS under review is part of a wider context of events. However, they are already proven, they only create a complement to the screening.

The complement or complementary "storyline" in a dream is a follow-up PS, and it is all examined in two states - waking and dream. Formally correct WG will generate the right activities to complement WG.

If this add-on creates the same activity in a dream as it does in "waking", it is a true PS from an object's perspective. These are used to determine the truth of the investigated PS.

\section{Conclusion}

Seemingly unrelated concepts such as dream, axiom and existence, sounding somewhat surrealistic, form one of the pillars of the definition and functioning of living objects. For example, the very role of a dream is seen in humans as an undesirable concomitant activity of 'peaceful sleep'. However, model calculations show that even the dream content, its wildness (!) Carries some information, and that ultimately the "prediction" of dreams has or at least may contain a small rational core $(-)$.

The level of ferocity is an indicator of the extent to which the PS needs to be rebuilt and is therefore to some extent indicative of what changes, or at least in what areas, the object can or should expect.

\section{References}

1. Vlcek M (2014) Would you like a GDP with your coffee?. Journal of Economics and Public Finance p. 2.

2. Vlcek M (2015) The Cell and its Worry about the Water. Journal of Cytology \& Histology 3: 1.

3. Vlcek M (2015) Relations among American Firms Allow the Rise of a New Object. American Journal of Economics 5: 423-427.

4. Vlcek M (2015) Structure of the Control of the World, published by Scholars' Press.

5. Vlcek M (2017) Dark Matter in our Ordinary Life, in Amazon - Wroclaw; ISBN 9781718882836 .

Copyright: (C2019 Vlček M. This is an open-access article distributed under the terms of the Creative Commons Attribution License, which permits unrestricted use, distribution, and reproduction in any medium, provided the original author and source are credited. 